\title{
DOTA: A Double Truthful Auction for Spectrum Allocation in Dynamic Spectrum Access
}

\author{
Qinhui Wang*, Baoliu Ye*, Tianyin $\mathrm{Xu}^{\dagger}$, Sanglu $\mathrm{Lu}^{*}$, and Song $\mathrm{Guo}^{\ddagger}$ \\ *National Key Lab. for Novel Software Technology, Nanjing University, China \\ ${ }^{\dagger}$ Department of Computer Science and Engineering, U.C. San Diego, USA \\ ${ }^{\ddagger}$ School of Computer Science and Engineering, University of Aizu, Japan
}

\begin{abstract}
Spectrum auctions have been proposed as an effective approach to fairly and efficiently trade the scarce spectrum resource among wireless users. The most significant challenge of the auction design to provide economic robustness, particularly truthfulness, under the local-dependent interference constraints. However, existing designs either do not consider spectrum reuse or are based on the impractical assumption that each user requests at most one channel. In this paper, we address this problem by proposing DOTA, a DOuble Truthful Auction for dynamic spectrum access. DOTA is economic-robust in terms of truthfulness, individual rationality, and no-deficit. It achieves improved utilization by exploiting spectrum reuse as well as dealing with the interference constraints. Moreover, DOTA minimizes the network transaction overhead and provides flexible channel bidding including range bidding and strict bidding.
\end{abstract}

\section{INTRODUCTION}

Radio spectrum is a critical but scarce resource for wireless communications. Usage of spectrum has long been governed by government agencies (e.g., FCC in USA, Ofcom in UK) who allocate spectrum by assigning licenses. Only licensed wireless users are allowed to use the spectrum. This allocation is in a relative long term with space-time invariance. On the other side, it has been widely recognized that the spectrum is becoming increasingly crowded under the long-term and exclusive management policy. However, most of the licensed spectrum is under-utilized [1], which desires a new policy trend [2] to make spectrum access dynamic.

With the advances in cognitive radio techniques, Dynamic Spectrum Access (DSA) was proposed to address the above dilemma [3], [4]. Under DSA, licensed users (called primary users) are encouraged to open up their idle spectrum to unlicensed users (called secondary users). In this way, primary users can obtain financial gains by leasing their idle spectrum. Auctions are widely accepted as an efficient approach to redistribute spectrum among users due to the perceived fairness and allocation efficiency [5]. The critical property required to design an auction is truthfulness, which ensures that each buyer's profits (called utility) could be maximized only by independently declaring his true valuation of the goods without knowing the bidding strategies of other bidders. Untruthful auctions are extremely vulnerable to market manipulation and may produce poor outcomes. Moreover, truthful auctions make life easier for bidders. Otherwise, each buyer has to acquire (or guess) the others' bidding strategies before being able to figure out a sophisticated optimal bidding strategy for himself.
Different from the conventional FCC-style auctions which include only a few large corporations located over national level regions, a typical auction for dynamic spectrum access consists of a large number of buyers distributed over a relatively small geographical region. Therefore, spectrum reusability can be exploited to significantly improve spectrum utilization, which makes it different from the conventional goods auctions, e.g., painting auctions. As a result, the reusability of spectrum makes the design of economic-robust (e.g., truthful) auctions more challenging [7], [8].

Most previous truthful designs for spectrum allocation (e.g., [8], [9], [10]) focus on single-sided buyer-only auctions under the assumption that sellers always trust the central authority. Nevertheless, sellers in reality are usually selfish and actively participate in auctions. Thus, the double auction is required for spectrum allocation. Unfortunately, most traditional truthful double auctions [11], [12] do not consider reusability. As a result, they either cannot be used for spectrum reuse or may lose truthfulness when applied to double spectrum auctions. TRUST [13] is the truthful double spectrum auction which achieves economic robustness while enabling spectrum reuse. However, TRUST is based on the "single channel request" assumption, i.e., each buyer requests at most one channel, which makes it impractical because bidding for multiple channels is more common in the real life.

In this paper, we propose DOTA, a DOuble Truthful spectrum Auction that enables spectrum reuse while achieving the economic robustness including truthfulness, individual rationality, and no-deficit, which is guaranteed by its clearing and pricing design. Moreover, DOTA supports flexible spectrum bidding (i.e., breaking TRUST's "single channel request" assumption) including range bidding and strict bidding. DOTA's clearing rule is able to translate the auction with multi-unit bids into an equivalent auction with single-unit bids. Further, the concept of translate-into-one bid [14] is introduced to minimize the network transactions so as to avoid additional transaction overhead (e.g., handoff overhead). We prove the economic-robustness of DOTA by theoretical analysis, and validate the effectiveness of DOTA using extensive simulations. The results show that DOTA can improve spectrum utilization by up to $61.4 \%$ while increasing the number of traded channels by up to $96 \%$, taking TRUST as a baseline. The improvement is mainly attributed to the trading quantity of channels, which benefit from the flexible bidding of bidders. 


\section{PReliminaries AND Related Work}

\section{A. Dynamic Spectrum Access}

In dynamic spectrum access networks, secondary users are allowed to dynamically share spectrum owned by primary users. In this way, user demands are more likely to be satisfied while the spectrum utilization can be improved. In this paper, we apply the Coordinated Dynamic Spectrum Access model [15] where a centralized spectrum broker periodically re-distributes the available spectrum from primary users to secondary users. Primary users (as sellers) selectively lease out their regional ownerships of spectrum channels by specifying ask prices. Meanwhile, secondary users (as buyers) bid for these available channels by specifying their bidding prices. Spectrum reusability can be highly exploited by addressing interference constraints. Moreover, to relieve the burden of market manipulation while simplifying the bidding strategies of bidders ${ }^{1}$, economic robustness (e.g., truthfulness) is critical to the design of the above auction-based framework.

\section{B. Economic-Robust Double Auction Mechanisms}

Double Auction Mechanisms. Different from single auctions, rational sellers also participate in the double auction. We describe the general double auction model as follows. In the first stage, the potential sellers $\mathcal{S}$ submit their ask prices $\mathbf{B}^{\mathbf{s}}$ to an auctioneer who simultaneously receives bids $\mathbf{B}^{\mathbf{b}}$ from the potential buyers $\mathcal{B}$. Then, the auctioneer generates marketclearing prices based on all bids and determines the winning sellers as well as the winning buyers. For a winning seller $j$, the market-clearing price $P_{j}^{s}$ is his actual payment received. For a winning buyer $i$, the clearing price $P_{i}^{b}$ is the price he pays. Let $V_{j}^{s}$ represent the true valuation of the auctioned goods of seller $j$, and let $V_{i}^{b}$ be the true valuation for requested goods of buyer $i$ that quantifies the benefit from the auction. The utility of a bidder is the gap between its benefit and his payment. Thus, the utility of seller $j$ is $U_{j}^{s}=P_{j}^{s}-V_{j}^{s}$, while the utility of buyer $i$ is $U_{i}^{b}=V_{i}^{b}-P_{i}^{b}$. We adopt the usual convention in economics, which assumes that bidders are rational and selfish in the sense that the goal of both sellers and buyers is to maximize their own utilities (i.e., profits).

Truthful Double Auction Mechanisms. Bidders may be selfish, i.e., they may declare deceptive valuation dishonestly, trying to increase their profits. To relieve the burden of market manipulation as well as the overhead of strategizing over others, truthfulness is required to be guaranteed for bidders. Truthful auctions ensure that bidders' utility will be maximized only by independently declaring their true valuation of the goods without knowing the other bidders' bids.

Definition 1 (Truthfulness): A double auction is truthful if and only if for any bidder $i$, its profit $U_{i}$ is maximized by bidding truthfully, i.e., $U_{i}\left(V_{i}\right) \geq U_{i}\left(B_{i}\right)$ for any $B_{i}$.

Besides truthfulness, Individual Rationality and No-Deficit are the other two important economic properties for economic robustness. The former guarantees non-negative utilities for

\footnotetext{
${ }^{1} \mathrm{~A}$ bidder is either a buyer or a seller.
}

bidders, thus providing the incentives of participation. The latter ensures the incentives of auctioneers to set up auctions.

Definition 2 (Individual Rationality): A double auction is individual rational if no winning seller is paid less than his bid and no winning buyer pays more than his bid, i.e., $P_{j}^{s} \geq B_{j}^{s}$ and $B_{i}^{b} \geq P_{i}^{b}$ for any seller $j$ and buyer $i$.

Definition 3 (No-Deficit): A double auction is No-Deficit if the auctioneer's profit is non-negative, i.e., $\phi \geq 0$, where $\phi$ is defined as the difference between the revenue collected from buyers and the expense paid to sellers:

$$
\phi=\sum_{i \in \mathcal{B}} P_{i}^{b}-\sum_{j \in \mathcal{S}} P_{j}^{s} \geq 0
$$

\section{Challenges of Double Spectrum Auction Design}

As mentioned above, the reusability of spectrum introduces significant challenges in achieving economic robustness. Conventional truthful double auctions (e.g., VCG [6], McAfee [11]) do not consider reusability. Moreover, as investigated in [13], prior reusability-driven single-sided auctions like VERITAS [8] may lose truthfulness when directly applied to double auctions. On the other hand, the Impossibility Theorem [18] shows that no double auction can simultaneously achieve all three economic properties (i.e., Definition 1, 2, 3) while maximizing auction efficiency. Thus, the auction design should firstly satisfy the economic properties and then approximately maximize efficiency. TRUST [13] is proposed to break the barrier between spectrum reusability and economic robustness. It is based on the "single channel request" assumption where each buyer requests at most one channel and each seller sells at most one channel. This assumption makes TRUST impractical since both buyers and sellers may request/sell multiple channels in reality. However, when allowing flexible spectrum bidding, the clearing rule and pricing rule have to be re-designed in order to guarantee economic robustness.

\section{Related Work}

Auctions have been widely used to allocate the scarce spectrum resource. How to efficiently allocate spectrum while enabling spectrum reuse has attracted great research interests. Gandhi et al., [16] propose a real-time spectrum auction framework which linearizes the interference constraints to achieve computational efficiency. Spectrum band auctions, e.g., [17], have also been studied. However, they do not take truthfulness into account. A number of truthful spectrum auctions [8], [9], [10] have been designed, but most of they only support singlesided auctions. Existing double auctions including single-unit [11], [12] and multi-unit [12], [14] provide truthfulness but do not exploit spectrum reusability. For example, the conventional double auction proposed in [12] may violate the property of no-deficit when applied to spectrum auction. The double spectrum auction proposed in [19] cannot address interference constraints which is of great importance to exploit spectrum utilization. TRUST [13], following the McAfee model [11], is the first truthful double auction for spectrum reusability. However, TRUST is a single-unit auction based on the "simple channel request" assumption. 


\section{DOTA AUCTION DESIGN}

In this section, we present the model for double spectrum auction problem and describe the detailed design of DOTA.

\section{A. Auction Model}

We consider a common spectrum trading scenario: multiple primary users (i.e., sellers) lease out their idle spectrum to other secondary users (i.e., buyers). Thus, non-conflicting secondary users can use the same channel simultaneously to improve utilization by exploiting spectrum reusability.

Assume that there are $m$ sellers and $n$ buyers participating in the auction. Each seller $j(1 \leq j \leq m)$ contributes $k_{j}$ distinct channels, and each buyer $i(1 \leq i \leq n)$ requests $d_{i}$ channels. The total $K=k_{1}+\cdots+k_{m}$ channels have uniform characteristics such that the requests of buyers are not channelspecific. We employ a conflict graph $G(V, E)$ [7] to model the conflict conditions. The vertex set $V$ contains $n$ vertices, with each vertex representing a buyer. In the edge set $E$, an edge indicates that two buyers will conflict with each other when they use the same channel at the same time.

We consider a single-round double spectrum auction with one auctioneer. We assume that bidders are rational and selfish. All bidders simultaneously submit their bids independently to the auctioneer without any knowledge of others, i.e., with no collusion. Let $B_{j}^{s}$ represent the bid submitted by seller $j$ for leasing one channel (i.e., per-channel price). $V_{j}^{s}$ is the true valuation of the channel. Similarly, $B_{i}^{b}$ and $V_{i}^{b}$ represent the bid and the true valuation of one channel for buyer $i$. After collecting all bids, the auctioneer determines the winners according to the clearing rule and charges each winner using the pricing rule. Let $P_{j}^{s}$ represent the actual payment of seller $j$ received from the auction and $P_{i}^{b}$ be the charged price from winning buyer $i$. Then, the utility of seller $j$ is $U_{j}^{s}=P_{j}^{s}-V_{j}^{s}$ if it wins the auction; otherwise, $U_{j}^{s}=0$. The utility of buyer $i$ is $U_{i}^{b}=V_{i}^{b}-P_{i}^{b}$ if it wins; otherwise, $U_{i}^{b}=0$.

\section{B. Design of DOTA}

The objective of DOTA is to design a double spectrum auction satisfying three economic properties: truthfulness, individual rationality, and no-deficit, which are critical to economicrobust double auctions. In a real auction market, there is a commission charge for each transaction. The situation is similar to network auctions because bidders also need to pay the transaction overhead, e.g., handoff overhead. Therefore, the bidders hope to trade with the least transactions to meet their demands/supplies. To minimize network transactions, we introduce the concept of translate-into-one bid [14], by which the high-demand buyers have more probability of winning the auction than the low-demand buyers. Translate-into-one bid can effectively increase the trading volume which is critical to improving spectrum utilization.

Define $C(k)$ as the transaction cost of trading $k$ channels at one time. For a certain number of channels, the cost is higher if more trades are required, i.e., $C\left(k_{1}+k_{2}\right)<C\left(k_{1}\right)+C\left(k_{2}\right)$. We define the translate-into-one price of buyer $i$ as follows:

$$
B_{i}^{*}=B_{i}^{b}+\frac{(k \times C(1)-C(k))}{k}
$$

where $k$ is the channel demand of buyer $i$.

We describe the detailed steps of DOTA as follows:

\section{Step I: Translate-into-one Bid}

Calculate the translate-into-one bid $B_{i}^{*}$ according to Equation (2). It is straightforward that $B_{i}^{*}>B_{i}^{b}$. Moreover, the higher demand of buyer $i$, the higher price $B_{i}^{*}$ that buyer $i$ will bid and thus have higher probability of winning according to DOTA's clearing rule (buyer $i$ will have the higher rank).

\section{Step II: Buyer Grouping Rule}

Assume that all the sellers' channels are available to all the buyers. DOTA organizes non-conflicting buyers into the same group to reuse channels. In the conflict model, the grouping is equivalent to finding the independent sets of the conflict graph. Note that the group formation can be easily extended to other interference models by using different grouping methods.

Let $G_{1}, G_{2}, \ldots, G_{L}$ represent the $L$ organized groups, and each group $G_{l}$ is treated as a single buyer (named group buyer) who bids per-channel price $\pi_{l}$ for requesting $\lambda_{l}$ channels. For any group $G_{l}$ with $n_{l}=\left|G_{l}\right|$ buyers, the group bid $\pi_{l}$ is:

$$
\pi_{l}=\min \left\{B_{i}^{*} \mid i \in G_{l}\right\} .
$$

The channel demand of the group $G_{l}$ is:

$$
\lambda_{l}=\max \left\{d_{i} \mid i \in G_{l}\right\} .
$$

\section{Step III: Clearing Rule}

In this step, DOTA determines auction winners. We sort the bids of both sellers and buyers in a decreasing order by the per-unit price. If a buyer and a seller have the same bid price, the buyer's bid is sorted into a higher rank. For the bidder with $k$-unit bids, the auctioneer translates the $k$-unit bids into $k$ single-unit bids. In other word, the auctioneer regards the bidder, e.g., group buyer $l$ or seller $j$, bidding $k$ channels as $k$ bidders each bidding 1 channel with per-channel price $\pi_{l}$ or $B_{j}^{s}$, respectively. The sorted bid set is denoted as follows:

$$
\{\cdots, \underbrace{\pi_{l}, \cdots, \pi_{l}}_{\lambda_{l}}, \cdots, \underbrace{B_{j}^{s}, \cdots, B_{j}^{s}}_{k_{j}}, \cdots\} .
$$

Let $b_{K+1}$ be the $(K+1)$-th bid in the ordered set given in Equation (5). Note that we only have at most $K$ group winners for total $K$ channels. DOTA supports flexible bidding requests including range requests and strict requests described as follows:

- Range Request. Each buyer will get $x$ channels where $x \in\left[0, d_{i}\right]$. DOTA determines the winners of range requests as follows. When the bidder of $b_{K+1}$ is a seller, the sellers with a bidding price ranked after the $(K+1)$ th position in the sorted bid set are winning sellers (including the $b_{K+1}$ seller). The buyers with a bidding price ranked before the $(K+1)$-th position in the sorted bid set are winning buyers. When the bidder of $b_{K+1}$ is a buyer, the winning sellers and the winning buyers are the same as above but excluding the bidder of $b_{K+1}$.

- Strict Request. Each buyer will get either all $d_{i}$ channels or nothing. DOTA determines the winners of strict requests as follows. The buyers with a bidding price more 
than $b_{K+1}$ are winning buyers. The winning sellers are chosen from sellers whose bidding price $b_{p} \leq b_{K+1}$. To ensure that the channels bought by buyers must be equal to that sold by sellers, we accordingly choose winning sellers from the one with low price to the one with high price satisfying $b_{p} \leq b_{K+1}$.

Toy Example. Consider an auction with 2 buyers and 4 sellers. Seller A sells 2 channels by bidding $\$ 6$ for each, seller B sells 1 channel by bidding $\$ 3$, and seller $\mathrm{C}$ sells 1 channel by bidding $\$ 2$. Buyer $\mathrm{D}$ needs 1 channels by bidding $\$ 5$, while buyer E needs 2 channels by bidding $\$ 4$ for each. Suppose that buyers $\mathrm{D}$ and $\mathrm{E}$ must use different channels, i.e., they are not in the same group. Then, the sorted bids set (c.f., Equation 5) is $\{6,6,5,4,4,3,2\}$. Notice that $K=4$ channels are available and the $(K+1)$-th bidder is buyer $\mathrm{E}$. If $\mathrm{D}$ and $\mathrm{E}$ use range requests, each of them wins 1 channel. Otherwise they use strict requests, only D wins 1 channel from seller C.

\section{Step IV: Pricing Rule}

When the bidder of $b_{K+1}$ is a buyer, $b_{K+1}$ is the clearing price. DOTA charges each buyer in the winning group the uniform price $b_{K+1}$ for each allocated channel. Meanwhile, DOTA pays each winning seller $b_{K+1}$ for each sold channel.

When the bidder of $b_{K+1}$ is a seller, $b_{K}$ is the clearing price. DOTA charges each buyer in the winning group $b_{K} \times d_{i}$ for strict requests and $b_{K} \times x$ for range requests. Meanwhile, the winning seller is paid the price $b_{K}$ for each sold channel.

\section{ECONOMIC ROBUSTNESS ANALYSIS}

We theoretically analyze the economic properties of DOTA in terms of truthfulness, individual rationality, and no-deficit.

Truthfulness. We prove the truthfulness of DOTA by showing the monotonicity of the clearing rule (Lemmas 1 and 2) and the bid-independence of the pricing rule (Lemmas 3 and 4).

Lemma 1: Given the buyers' bid set $\left\{B_{k}^{b}\right\}_{k=1, k \neq i}^{n}$ and the sellers' bid set $\left\{B_{j}^{s}\right\}_{j=1}^{m}$, if buyer $i$ wins the auction by bidding $B_{i}^{b}$, buyer $i$ can also win by bidding $\tilde{B}_{i}^{b}$, where $\tilde{B}_{i}^{b}>B_{i}^{b}$.

Proof: Let $\pi_{l}$ and $\tilde{\pi}_{l}$ be the bid of group $l$ that buyer $i$ belongs to when buyer $i$ bids $B_{i}^{b}$ and $\tilde{B}_{i}^{b}$ respectively. Since $\tilde{B}_{i}^{b}>B_{i}^{b}$, we have $\tilde{\pi}_{l} \geq \pi_{l}$. If $\tilde{\pi}_{l}=\pi_{l}$, our claim holds. If $\tilde{\pi}_{l}>\pi_{l}$, the position of group $l$ with $\tilde{\pi}_{l}$ is sorted before group $l$ with $\pi_{l}$ in Equation (5), because the bid set is sorted in the decreasing order. Therefore, buyer $i$ will win in both groups according to the clearing rule.

Lemma 2: Given the buyers' bid set $\left\{B_{i}^{b}\right\}_{i=1}^{n}$ and the sellers' bid set $\left\{B_{k}^{s}\right\}_{k=1, k \neq j}^{m}$, if seller $j$ wins by bidding $B_{j}^{s}$, $j$ can also win by bidding $\tilde{B}_{j}^{s}$, where $\tilde{B}_{j}^{s}<B_{j}^{s}$.

Proof: This proof is similar as Lemma 1.

Lemma 3: Given the buyers' bid set $\left\{B_{k}^{b}\right\}_{k=1, k \neq i}^{n}$ and the sellers' bid set $\left\{B_{j}^{s}\right\}_{j=1}^{m}$, if buyer $i$ wins the auction by bidding $B_{i}^{b}$ or $\tilde{B}_{i}^{b}$, the clearing price charged from buyer $i$ is the same for both bids.

Proof: Let $\pi_{l}$ and $\tilde{\pi}_{l}$ be the bid of group $l$ that buyer $i$ belongs to when buyer $i$ bids $B_{i}^{b}$ and $\tilde{B}_{i}^{b}$, respectively. Since buyer $i$ wins in both groups, the clearing price is determined by a bid ranked after the bid of $B_{i}^{b}$ and $\tilde{B}_{i}^{b}$, which is the same in both groups, i.e., the clearing price is the $(K+1)$-th bid ranked after both $B_{i}^{b}$ and $\tilde{B}_{i}^{b}$ ). Thus, our claim holds.

Lemma 4: Given the buyers' bid set $\left\{B_{i}^{b}\right\}_{i=1}^{n}$, the sellers' bid set $\left\{B_{k}^{s}\right\}_{k=1, k \neq j}^{m}$, if seller $j$ wins the auction by bidding $B_{j}^{s}$ and $\tilde{B}_{j}^{s}$, the price paid to $j$ is the same for both bids.

Proof: This proof is similar as Lemma 3.

Having the above four lemmas, we prove that DOTA is truthful for both sellers and buyers.

Theorem 1: DOTA is truthful for sellers.

Proof: Let $V_{j}^{s}$ be the truthful bidding for seller $j$, and $\tilde{B}_{j}^{s}$ be the deceptive bidding where $\tilde{B}_{j}^{s} \neq V_{j}^{s}$. We have the following two cases ( $\mathrm{C} 1$ and $\mathrm{C} 2$ ) corresponding to whether seller $j$ wins or loses:

C1 Seller $j$ loses by bidding $\tilde{B}_{j}^{s}$, so his utility is 0 . If seller $j$ bids truthfully, his utility will be non-negative $(\geq 0)$.

C2 Seller $j$ wins by bidding $\tilde{B}_{j}^{s}$. If seller $j$ also wins by $V_{j}^{s}$, the utilities are the same because the payment of seller $j$ does not change (Lemma 4). Let us consider that seller $j$ loses by bidding $V_{j}^{s}$. Let $p_{j}$ and $\tilde{p}_{j}$ be the payment of the winning sellers when seller $j$ bids $V_{j}^{s}$ and $\tilde{B}_{j}^{s}$, respectively. Since seller $j$ wins by bidding untruthfully and loses by bidding truthfully, we have $\tilde{B}_{j}^{s}<V_{j}^{s}$ (Lemma 2). Moreover, the rank of price bid in the sorted bids set (i.e., Equation (5)) stays unchanged when seller $j$ lowers its bids, because the bids set are in a decreasing order. Hence, $\tilde{p}_{j}=p_{j}$ according to the pricing rule. Since seller $j$ wins by bidding $\tilde{B}_{j}^{s}$, we have $\tilde{B}_{j}^{s} \leq \tilde{p}_{j}$ (by the pricing rule). Similarly, we get $p_{j} \leq V_{j}^{s}$. As a result, $\tilde{B}_{j}^{s} \leq \tilde{p}_{j}=p_{j} \leq V_{j}^{s}$. Therefore, the utility of seller $j$ who bids untruthfully is $\left(\tilde{p}_{j}-V_{j}^{s}\right) \times N_{s} \leq 0$, where $N_{s}$ is the number of channels sold by seller $j$.

Thus, in both cases, bidding other than the truthful valuation is not better than bidding the truth valuation.

Theorem 2: DOTA is truthful for buyers.

Proof: Let $V_{i}^{b}$ be the truthful bidding for buyer $i$, and $\tilde{B}_{i}^{b}$ be the deceptive bidding where $\tilde{B}_{i}^{b} \neq V_{i}^{b}$. We also have the following two cases (C3 and $\mathrm{C} 4)$ :

C3 Buyer $i$ loses by bidding $\tilde{B}_{i}^{b}$, so his utility is 0 . If buyer $i$ bids truthfully, his utility will be non-negative $(\geq 0)$.

C4 Buyer $i$ wins by bidding $\tilde{B}_{i}^{b}$. If buyer $i$ also wins by $V_{i}^{b}$, the utilities are the same since the payment of buyer $i$ does not change (Lemma 3). Let us consider that buyer $i$ loses with $V_{i}^{b}$. Let $\pi_{l}$ and $\tilde{\pi}_{l}$ be the bid of buyer $i$ 's group when $i$ bids $V_{i}^{b}$ and $\tilde{B}_{i}^{b}$. Since buyer $i$ changes the auction results by bidding higher than $V_{i}^{b}$, the bid of buyer $i$ when bidding truthfully must be the lowest bid in the group, i.e., $\pi_{l}=V_{i}^{b}$. Moreover, since buyer $i$ loses by bidding $V_{i}^{b}$ and wins by bidding $\tilde{B}_{i}^{b}$, the inequality $\pi_{l} \leq p \leq \tilde{\pi}_{l}$ holds (Lemma 1), where $p$ is the clearing price charged from its group when it wins. Therefore, the utility when buyer $i$ bids $\tilde{B}_{i}^{b}$ is $\left(V_{i}^{b}-p\right) \times N_{b} \leq 0$, where $N_{b}$ is the number of channels bought by buyer $i$.

Thus, in both cases, bidding other than the truthful valuation is not better than bidding the truth valuation.

Having Theorem 1 and 2, we can directly get:

Theorem 3: DOTA is truthful for bidders. 


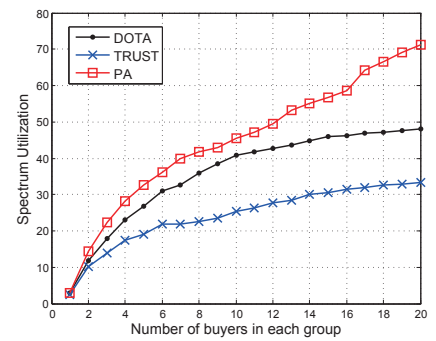

(a) Spectrum Utilization

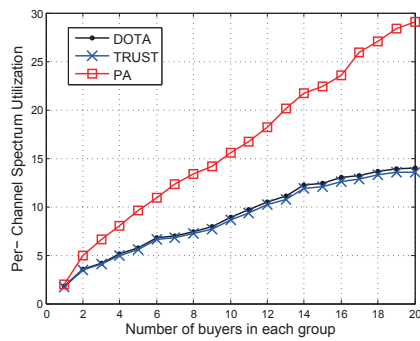

(b) Per-Channel Utilization

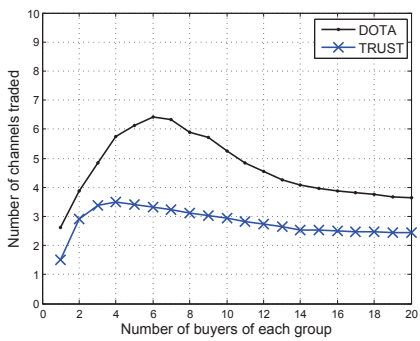

(c) Number of Traded Channels

Fig. 1. Compare DOTA with TRUST and PA in terms of (a) spectrum utilization, (b) per-channel utilization and (c) number of traded channels

Individual Rationality is proved by the following theorem:

Theorem 4: DOTA is individual rational, i.e., no winning seller is paid less than his bid and no winning buyer is charged more than his bid.

Proof: Let $b_{p}$ be the clearing price $\left(b_{K+1}\right.$ or $\left.b_{K}\right)$. Firstly, since the bids of sellers are in the non-decreasing order and DOTA pays each winning seller $j$ with $b_{p}$ where $b_{p} \geq B_{j}^{s}$, we have $B_{j}^{s} \leq b_{p}=P_{j}^{s}$, i.e., no winning seller is paid less than his bid. Secondly, for each winning buyer $i$ in group $G_{l}, G_{l}$ 's bid $\pi_{l}$ must be no less than $b_{p}$ because bids are sorted in the non-increasing order. Thus, we have $P_{i}^{b}=b_{p} \leq \pi_{l} \leq B_{i}^{b}$, i.e., no winning buyer is charged more than his bid.

Non-Deficit is proved by the following theorem:

It is easy to show that the pricing rule of DOTA ensures that the auction will clear when each round ends [14].

Lemma 5: DOTA clears the auction, i.e., the total number of channels sold by sellers is equal to that bought by buyers.

Theorem 5: DOTA is no-deficit, i.e., $\phi \geq 0$.

Proof: According to the pricing rule of DOTA, the clearing price for winning bidders (sellers or buyers) are the same. Let $b_{p}$ be the clearing price $\left(b_{K+1}\right.$ or $\left.b_{K}\right)$, the revenue collected from buyers is $b_{p} \times N_{b}$ where $N_{b}$ is the total number of channels bought by buyers, while the expense paid to sellers is $b_{p} \times N_{s}$ where $N_{s}$ is the total number of channels that sold by sellers. Therefore $\phi=b_{p} \times N_{b}-b_{p} \times N_{s}=0$ (Lemma 5).

\section{Performance Evaluation}

We use extensive simulations to evaluate the performance of DOTA, compared with other double auction designs. Further, we study the impact of grouping methods, as well as the performance gain of the translate-into-one bid mechanism.

\section{A. Simulation Methodology}

We deploy bidders in a $1 \times 1$ square with 10 sellers and 100 buyers randomly deployed. We assume that the bid of each bidder (seller or buyer) is randomly distributed within $(0,1]$. Each seller contributes one channel and the demand of each buyer is randomly set as 1,2 , or 3 . To mitigate the effect of randomness, the results are averaged over 5 random seeds, with each running 100 rounds. We use the following performance metrics:

1) Spectrum Utilization is represented by the total number of winning buyers.
2) Per-channel Utilization is the total number of buyers who obtain the same channel.

3) Number of Traded Channels is the total number of channels that have been allocated.

\section{B. Simulation Results}

Fig. 1 shows the performance of DOTA in terms of spectrum utilization under the default setting, compared with TRUST [13] and PA (Pure Allocation). In TRUST, each buyer requires only one channel, while PA always chooses the group with the largest size, aiming at maximizing spectrum utilization without truthfulness guarantee. We let the number of channels in PA be equal to the number of traded channels in DOTA for a fair comparison. PA has the highest spectrum utilization beyond DOTA and TRUST. It is known that making auctions economic robustness always lead to degradation on spectrum utilization [13], [10]. Fig. 1(a) shows that DOTA achieves higher overall spectrum utilization with the increasing number of per-group buyers, compared with TRUST. Fig. 1(b) shows that DOTA and TRUST have similar per-channel utilization, which indicates the improvement of spectrum utilization by DOTA is mainly attributed to the increased number of traded channels. Fig. 1(c) shows the number of traded channels in DOTA and TRUST. The improvement of trading quantity is due to the the flexible bidding of DOTA, which allows each buyer to bid for multiple channels.

\section{Discussion on Grouping Methods}

Since the grouping mechanism is a key component of DOTA, we extensively study three grouping methods on DOTA [13], i.e., MAX-IS, Greedy, and Random.

- Max-IS recursively finds the maximum independent set of the conflict graph to form a group.

- GREEDY recursively chooses a node with the minimal degree based on the conflict graph to form a group.

- RANDOM randomly chooses some non-conflicting nodes to form a group.

We limit the scale of each group as no more than 8, i.e., at most 8 buyers may reuse the same channel. We examine them under the following different network topologies with same numbers of bidders deployed:

- Random Topology which is the default network setting with an average conflict degree (i.e., the degree of conflict graph) of 7.3 on average. 


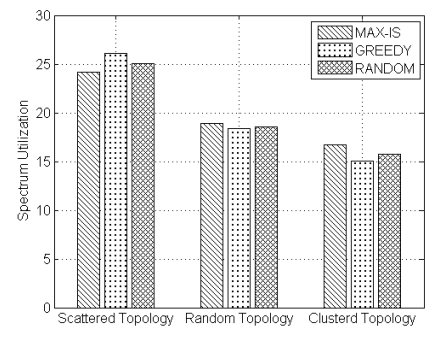

Fig. 2. The impact of different grouping methods on DOTA

- Cluster Topology where buyers are gradually placed in a small area so as to create hotspots with the conflict degree being around 20.4 on average.

- Scatter Topology where larger interference range is set, leading to low average degree which is around 2.4.

Fig. 2 shows the impact of different grouping methods on DOTA in terms of spectrum utilization. According to the figure, Max-IS achieves higher utilization than GREEDY and RANDOM under the cluster topology, while GREEDY achieves the highest utilization under the scatter topology. Under the random topology, the utilization of the three methods is similar. Since a group bid is the minimum one of buyers in the group, a larger group size yields smaller group bid but generates high spectrum reuse. Intuitively, the group size in cluster topology is less than the one in scatter topology because of the conflicts. In cluster topology where the group size is small and diverse, MAX-IS increases the spectrum utilization because of choosing relatively large group. In scatter topology with large group size, GREEDY chooses relatively small group with high bid, therefore achieving high spectrum utilization. In summary, the group method should be carefully chosen in consideration of various network topologies.

\section{Impact of Translate-into-one Bid}

Fig. 3 shows the effect of the translate-into-one bid in DOTA. We can see that DOTA achieves higher spectrum utilization when using the translate-into-one bid compared with the one using the original bid. With the increase of the group size, especially after reaching 10 buyers per group, the effect of translate-into-one bid is getting marginal. This is because the differences of the channel demands of groups are becoming smaller with more group buyers, since the group demand is the maximum demand of the group buyers.

\section{CONCLUSION}

We have proposed DOTA, a double spectrum auction, which achieves economic robustness while enabling spectrum reuse. DOTA provides flexible bidding requests by translating multiunit bids into equivalent single-unit bids. Moreover, DOTA minimizes the additional transaction overhead by the translateinto-one bid mechanism. We show that DOTA improves spectrum utilization up to $61.4 \%$ and the number of traded channels up to $96 \%$, compared with the state-of-the-art double auction.

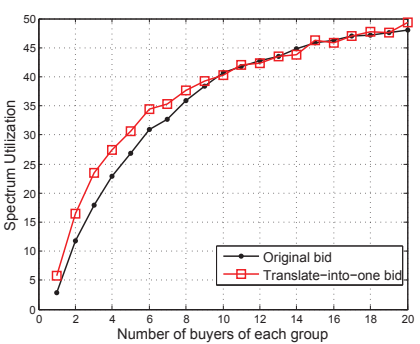

Fig. 3. Impact of translate-into-one bid on spectrum utilization

\section{ACKNOWLEDGEMENTS}

This work is partially supported by the National Natural Science Foundation of China under Grant No. 60903025, 61170069, 60903173, and 60721002; the National Basic Research Program of China (973) under Grant No. 2009CB320705; the scientific support program of Jiangsu province under Grant No. E2010179.

\section{REFERENCES}

[1] FCC Spectrum Policy Task Force, "Report of the Spectrum Efficiency Working Group", Nov. 2002. Available at: http://www.fcc.gov/sptf/reports.html.

[2] Spectrum Policy Task Force, "Spectrum Policy Task Force Report", Federal Communications Commission ET Docket 02-135, 2002.

[3] R. J. Berger, "Open Spectrum: A Path to Ubiquitous Connectivity", ACM Quеие 1(3), 2003.

[4] I. Akyildiz, W. -Y. Lee, M. Vuran, and S. Mohanty, "NeXt generation/dynamic spectrum access/cognitive radio wireless networks: a survey", Computer Networks, 50:2127-2159, 2006.

[5] P. Klemperer, "Auctions, theory and practice", Princeton University Press, 2004.

[6] M. Babaioff and N. Nisan, "Concurrent auctions across the supply chain", In Proc. of ACM Conference on Economic Commerce (EC), 2001.

[7] K. Jain, J. Padhye, V. N. Padmanabhan, and L. Qiu, "Impact of interference on multi-hop wireless network performance", In Proc. of ACM MobiCom, 2003.

[8] X. Zhou, S. Gandhi, S. Suri, and H. Zheng, "eBay in the Sky: StrategyProof Wireless Spectrum Auctions", In Proc. of ACM MobiCom, 2008.

[9] J. Jia, Q. Zhang, Q. Zhang, and M. Liu, "Revenue Generation for Truthful Spectrum Auction in Dynamic Spectrum Access", In Proc. of ACM MobiHoc, 2009.

[10] Q. Wang, B. Ye, T. Xu, and S. Lu, "An Approximate Truthfulness Motivated Spectrum Auction for Dynamic Spectrum Access", In Proc. of IEEE WCNC, 2011.

[11] R. P. McAfee, "A dominant strategy double auction". Journal of Economic Theory, 56(2): 434-450, 1992.

[12] K. Deshmukh, A. Golddberg, J. Hartline, and A. Karlin, "Truthful and Competitive Double Auctions", In Proc. of Annual European Symposium on Algorithms (ALGO), 2002.

[13] X. Zhou and H. Zheng, "TRUST: A General Framework for Truthful Double Spectrum Auctions", In Proc. of IEEE INFOCOM, 2009.

[14] H. Yin, X. Jia, and D. Fang, "A Novel Double Auction Mechanism for Electronic Commerce: Theory and Implementation", In Proc. of International Conference on Machine Learning and Cybernetics, 2004.

[15] M. Buddhikot, P. Kolodzy, S. Miller, K. Ryan, and J. Evans, "DIMSUMnet: New directions in wireless networking using coordinated dynamic spectrum access", In Proc. of IEEE WoWMoM, 2005.

[16] S. Gandhi, C. Buragohain, L. Cao, H. Zheng, and S. Suri, "A General Framework for Wireless Spectrum Auctio", In Proc. of DySPAN, 2007.

[17] M. Buddhikot and K. Ryan, "Spectrum management in coordinated dynamic spectrum access based cellular networks", In Prof. of IEEE DySPAN, 2005.

[18] R. B., Myerson and A. M. Satterthwaite, "Efficient mechanisms for bilateral trading", Journal of Economic Theory, 29:265-281, 1983.

[19] H. Xu, J. Jin and B. Li, "A Secondary Market for Spectrum", In Proc. of IEEE INFOCOM, 2010 